\title{
1 A functional hierarchy for choice in medial prefrontal cortex
}

David J-N. Maisson*, Tyler V. Cash-Padgett, Benjamin Y. Hayden, Sarah R. Heilbronner, and Jan Zimmermann

Department of Neuroscience, Center for Magnetic Resonance Research, Center for

Neuroengineering Department of Biomedical Engineering

University of Minnesota, Minneapolis MN 55455

\section{* Corresponding author}

David J-N Maisson

Department of Neuroscience,

University of Minnesota

Minneapolis, MN, 55455

20

Email: maiss002@umn.edu

\section{Keywords}

anterior cingulate cortex, medial wall, pregenual, subgenual, ventromedial prefrontal cortex

\section{Acknowledgements}

We thank Meghan Castagno Pesce, Marc Mancarella, Caleb Strait and Tommy Blanchard for assistance with data collection, and the rest of the Hayden/Zimmermann lab for valuable discussions. This research was supported by a National Institute on Health grant R01 DA038106 (to BYH) a R01 MH118257 (to SRH), a National Institute on Drug Abuse grant P30 DA04874201A1 (to BYH, SH and JZ), a National Institute for Biomedical Imaging Grant P41 EB027061 (to BYH and JZ), and a UMN AIRP award (to BYH, SH and JZ).

\section{Competing interests}

The authors have no competing interests to declare. 

Hierarchical approaches to functional neuroanatomy propose that choice-relevant brain

39 regions have overlapping functions and can be organized into a series that progressively

40 transforms information about options into choices. Here, we examined responses of neurons in

41 four regions of the medial prefrontal cortex as macaques performed two-option risky choices. All

42 four regions encoded economic variables in similar proportions and showed putative signatures

43 of key choice-related computations. We found evidence for a hierarchical organization

44 proceeding from areas $14 \rightarrow 25 \rightarrow 32 \rightarrow 24$. Specifically, we found that decodability of eight

45 distinct task variables increased along that path, consistent with the idea that hierarchically later

46 regions make these variables more separable. We also found longer intrinsic timescales in the

47 same series, further supporting the idea of a hierarchy. Together these results highlight the

48 importance of the medial wall in choice, endorse a specific hierarchical organization, and argue

49 against a modular functional neuroanatomy of choice. 
Economic choice is mediated by a large number of brain regions, including several in the

54 prefrontal cortex (Rangel et al., 2008; Haber and Knutson, 2010; Rushworth et al., 2011; Haber

55 and Beherens, 2014). The principles delineating the parcellation of function in choice remain

56 unclear. On one hand, modular theories hold that specific brain regions can be associated with

57 particular nameable functions, such as evaluation, comparison, and action selection (Rangel et

58 al., 2008; Kable and Glimcher, 2009; Padoa-Schioppa, 2011; Passingham and Wise, 2015;

59 Hauser et al., 2015; Hunt et al., 2018). On the other hand, distributed approaches to

60 understanding choice hold that particular elements of choice do not correspond neatly to

61 anatomical regions (Cisek and Kalaska, 2010; Cisek, 2012; Hunt and Hayden, 2017; Yoo and

62 Hayden, 2018). Distributed approaches are inspired by classic connectionist theories as well as

63 by modern deep learning approaches (Plaut, 1995; Bengio et al., 2003; Pennington et al., 2014;

64 Hunt and Hayden, 2017; Balasubramani et al., 2018). They are also inspired by cognitive and

65 philosophical theories of distributed cognition (Uttal et al., 2001; Prinz, 2006; Anderson, 2007),

66 and by analogy to the form vision system, where hierarchical models have come to replace

67 historically dominant modular models (Maunsell and van Essen, 1983; DiCarlo et al., 2012; Yoo

68 and Hayden, 2018).

69 Within the domain of distributed models, early mass-action and equipotentiality models

70 have lately been supplanted by hierarchical distributed models (Fuster, 1990; Fellemen and Van

71 Essen, 1991; Rushworth et al., 2011). We and others have extended the approach by proposing

72 one described by a gradual transformation of information, but that networked areas show graded

73 functional distinctions (Hunt and Hayden, 2017; Hunt et al., 2012; Hunt et al., 2015). For

74 example, specific circuits within the prefrontal cortex may be organized into a hierarchical 
75 gradient, so that each anatomical region implements part of a transformation of task-relevant

76 information from a domain of options to a domain of actions. We have proposed that each region

77 untangles information about the best action, which is latent in the early representations and

78 which, through serial processing, is transformed into appropriate choice actions (Yoo and

79 Hayden, 2018). This view is inspired by, and is an extension of, modern untangling-based

80 models of form vision (DiCarlo and Cox, 2007; DiCarlo et al., 2012).

81 A critical prediction of hierarchical models is that it should be possible to arrange medial

82 prefrontal regions into a particular hierarchical organization on the basis of their functional

83 properties. Discussions of prefrontal hierarchy have typically focused on the lateral surface, or,

84 when examining the medial wall, on the rostrocaudal axis (Koechlin et al., 2002; Rushworth et

85 al., 2007; Badre, 2008; Nee et al., 2013; Fuster, 2001; Cai and Padoa-Schioppa, 2014; Siegel et

86 al., 2015). We were interested, instead, in the ventrodorsal dimension of the medial prefrontal

87 cortex. Neuroeconomists have long proposed that the orbitofrontal cortex serves as the entry

88 point of choice-relevant sensory information into the prefrontal cortex and that the motor cortex

89 serves as the output (e.g. Hare et al., 2011; Padoa-Schioppa, 2011; Rudebeck and Murray, 2014;

90 Murray et al., 2014). The medial wall inferior to the premotor cortex, which includes areas 14,

9125,32 , and 24 , looks to be a likely pathway linking OFC to pre/motor areas. These regions also

92 have prominent limbic, visceral, and reward-related functions, suggesting they may contribute to

93 valuation and perhaps to choice (Vogt et al., 1979; Carmichael and Price, 1995, 1996; Freedman

94 et al., 2000).

However, there are several possible functional hierarchies that are consistent with known

96 anatomy. First, it could follow topology in a rough ventrodorsal direction $(14 \rightarrow 25 \rightarrow 32 \rightarrow 24)$.

97 Second, it could match the contour of the genu of the corpus callosum $(25 \rightarrow 14 \rightarrow 32 \rightarrow 24)$. Third, 
98 cytoarchitecture suggests that the less differentiated cingulate areas $(25,32$, and 24$)$ may precede

99 the more differentiated area 14 (Barbas and Pandya, 1989). Other cytoarchitectural studies

100 suggest that pre- and subgenual regions (14, 25, and 32) may have shared function but differ

101 qualitatively from the postgenual 24 (Bush et al., 2000). These specific pathways have not, to our

102 knowledge, been functionally evaluated. Despite this, identifying the functional hierarchy, if one

103 exists, is critical for establishing a neuroscience of economic choice (Rangel et al., 2008).

104 We examined a composite dataset consisting of single unit responses collected in these

105 four brain regions, including previously published data for 14, 25, and 24, and newly collected

106 data for area 32 (Strait et al., 2014; Azab and Hayden, 2017, 2018). Instead of looking for

107 specific functions that would distinguish these regions from each other, we took a function-first

108 approach: we selected key putative signatures of participation in specific elements of choice, and

109 then characterized each region in these functions. Overall, we found two major results. First, the

110 regions all show signatures of all tested functions and do not differ qualitatively in whether they

111 carry certain information or have signatures of choice processes. Second, a decoding analysis

112 shows both stronger decodability on eight dimensions consistent with a single hierarchy,

113 specifically one that progresses from $14 \rightarrow 25 \rightarrow 32 \rightarrow 24$. This second result is complemented by a

114 demonstration that intrinsic timescale shows the same pattern. Together these results support a

115 specific ventrodorsal medial prefrontal hierarchy and, simultaneously, argue against a modular

116 view in which conceptually distinct functions are reified in neuroanatomy. 


\section{RESULTS}

\section{Behavioral results}

Rhesus macaque (Macaca mulatta) subjects performed one of two structurally similar

121 two-alternative forced choice gambling tasks (Strait et al., 2014; Azab and Hayden, 2017; see

122 Methods). Briefly, subjects chose between two risky options presented asynchronously (Figure

123 1A-B). After the second offer was presented, a go cue indicates that the subject was free to shift

124 gaze toward the target option to indicate a choice. Thus, the task was naturally divided into three

125 epochs, corresponding to the periods immediately following offer 1 , offer 2 , and choice. The

126 offer 2 epoch is the first during which the subject can compare subjective values and select an

127 action. (Note, however, that subjects can and likely do form tentative partial choices based solely

128 on the value of the first offer, Azab and Hayden, 2017).

A

offer 1

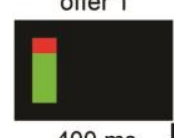

delay 1

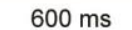

chance of NO reward $(0 \mu \mathrm{L})$

chance of SMALL reward $($ mean $=125 \mu \mathrm{L}$ )

chance of MEDIUM reward (mean $=165 \mu \mathrm{L}$ )

chance of LARGE reward $($ mean $=240 \mu \mathrm{L})$

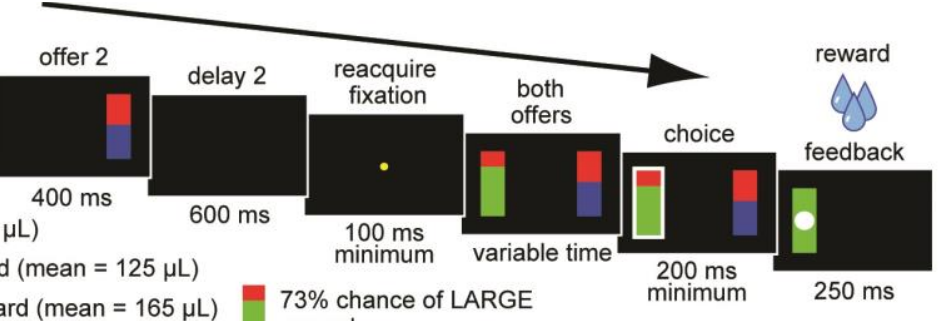

reward

B

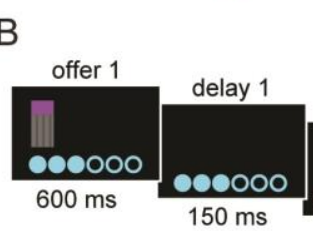

chance of -2 tokens

chance of -1 token

chance of no token change

chance of +1 token

$52 \%$ chance of MEDIUM

reward

chance of +2 tokens

chance of +3 tokens

131 Figure 1. Gambling Tasks. A. Risky choice task: The first offer is presented, followed by a delay period, after which

132 the second offer is presented. After another delay period, fixation is reacquired for a minimum of $100 \mathrm{~ms}$. Both offers 
133 are then presented, a choice is made, and the choice is probabilistically rewarded. Offers consist of a reward

134 magnitude (color of the non-red portion of the bar) and a probability (size of the colored portion). B. Token risky

135 choice task: Equivalent format but reward is tokenized. Once tokens reach 6, a reward is delivered.

Behavior in these tasks has been explored at length and is not reanalyzed here (for the

138 most detailed analyses, see Farashahi et al., 2018; Farashahi et al., 2019). Briefly, behavior

139 reflected understanding of all important task variables with very weak order or side biases. We

140 defined the expected value of an offer as the product of the offer magnitude (in uL juice) and

141 probability of reward. Thus, for a basic characterization of behavior, we computed the frequency

142 with which a given offer was chosen when it had a higher expected value. We determined the

143 proportion of trials on which the subject chose the first offer and we compared it to the

144 difference in expected values of the two offers for each trial. Subjects' behavior described a

145 sigmoidal function (Figure 2A). Subjects most frequently chose the offer with the higher

146 expected value (vmPFC sessions: $84.55 \%$ of trials; sgACC sessions: $78.62 \%$; pgACC sessions:

147 74.97\%; dACC sessions: 75.57\%; $p<0.0001$ in all cases; 1 -sample t-test), consistent with the

148 idea that they had a basic understanding of the task. 
A

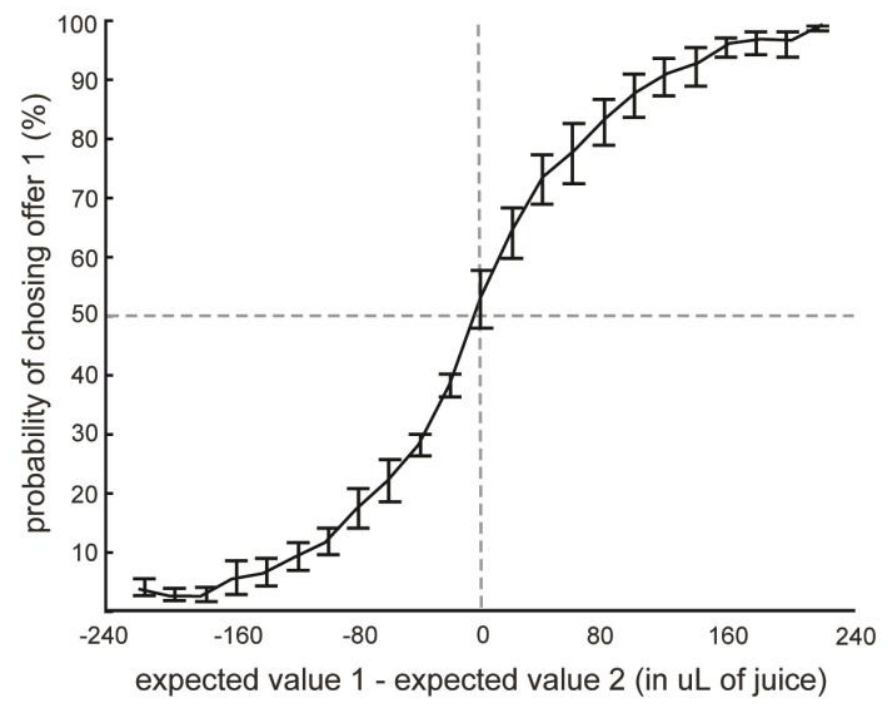

B

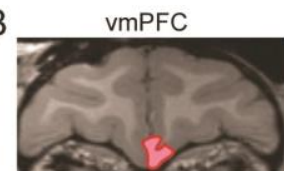
$\operatorname{sgACC}$
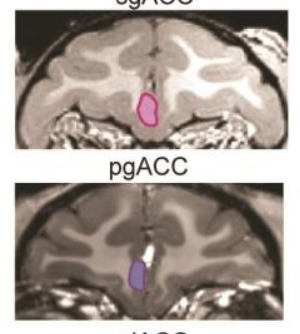

dACC

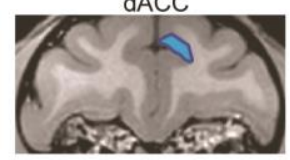

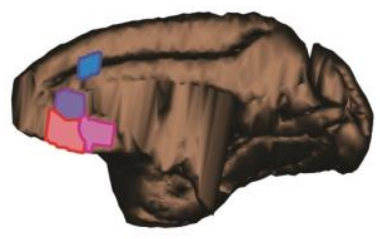

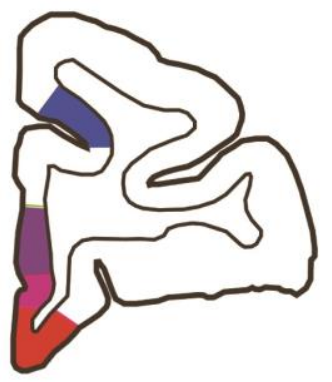

Figure 2. Target brain areas and behavior. A. Average behavior across all subjects and recording sessions. Error

bars reflect the standard error across sessions. B. MRI scans of the brain areas targeted for recordings. A single

representative subject is chosen for each area even though two subjects were recorded for each. The right panels

denote the targeted brain areas on both transverse and coronal planes.

Firing rates in all regions encode values of offers

We recorded neuronal activity from four brain regions: ventromedial prefrontal area 14

(vmPFC), subgenual anterior cingulate area 25 (sgACC), pregenual anterior cingulate area 32

previously published. Some data from vmPFC, sgACC, and dACC have been previously

published, although the key analyses here are all new (Azab and Hayden, 2017, 2018, and 2020;

Strait et al., 2014, 2015, and 2016). We collected these recordings from 4 subjects (B, H, V \& J

see Methods and Figure 2B). For each area, we recorded from two subjects, although we did 
collected data from $\mathrm{dACC}$ and $\mathrm{sgACC}$ in the token risky choice task; we collected data from vmPFC and pgACC in the risky choice task (Figure 1A-B). The basic format for each of the tasks during the selected time period for analysis, from within each trial, are essentially the same.

169 We do not believe the small differences between the two tasks influenced the results we present 170 here.

First, we confirmed our hypothesis that there would be no differences between areas in

174 stimulus (i.e. the offer) response latencies. For both offer 1 and offer 2, we computed the latency

175 of neural responses (see Methods). We defined latency as the time elapsed from the onset of the

176 offer stimulus until firing rates in the respective epoch reached their maximum within the epoch.

177 We used a 4 (area) x 2 (offer 1 or 2) ANOVA to test for differences. Neither the main effect of

178 area $(\mathrm{F}=1.96, p=0.297)$ nor offer number $(\mathrm{F}=5.04, p=0.110)$ was statistically significant

179 (Figure 3A). Neuronal responses to the onset of offer 1 reached maximum firing (spikes per

180 millisecond) in epoch 1 after an average of $258.55 \mathrm{~ms}$ from the offer onset (vmPFC $=265.6 \mathrm{~ms}$;

$181 \operatorname{sgACC}=242.5 \mathrm{~ms} ; \mathrm{pgACC}=252 \mathrm{~ms} ; \mathrm{dACC}=274.1 \mathrm{~ms})$. Neuronal responses in epoch 2

182 reached their maximum, on average, $245.04 \mathrm{~ms}$ after the onset of offer $2(\mathrm{vmPFC}=241.8 \mathrm{~ms}$;

$183 \operatorname{sgACC}=242.2 \mathrm{~ms} ; \operatorname{pg} \mathrm{ACC}=245.6 \mathrm{~ms} ; \mathrm{dACC}=250.5 \mathrm{~ms})$. 


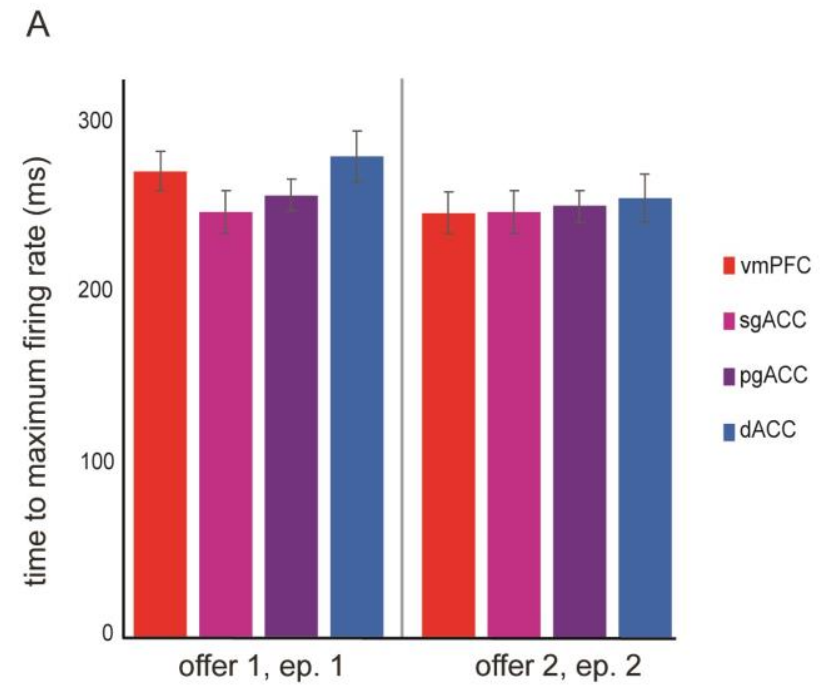

We examined the proportion of neurons in each region selective for the value of offer 1 during epoch 1. Figure 3B-E shows the average firing rate responses of an example neuron from each of the four regions. In all four regions, a significant proportion of neurons encoded the value of offer 1 during the offer 1 epoch (vmPFC: $16.03 \%$; sgACC: 10.27\%; pgACC: 10.98\%; dACC: $26.36 \% ; p<0.01$ in all areas, binomial test). Likewise, neurons in three of the four

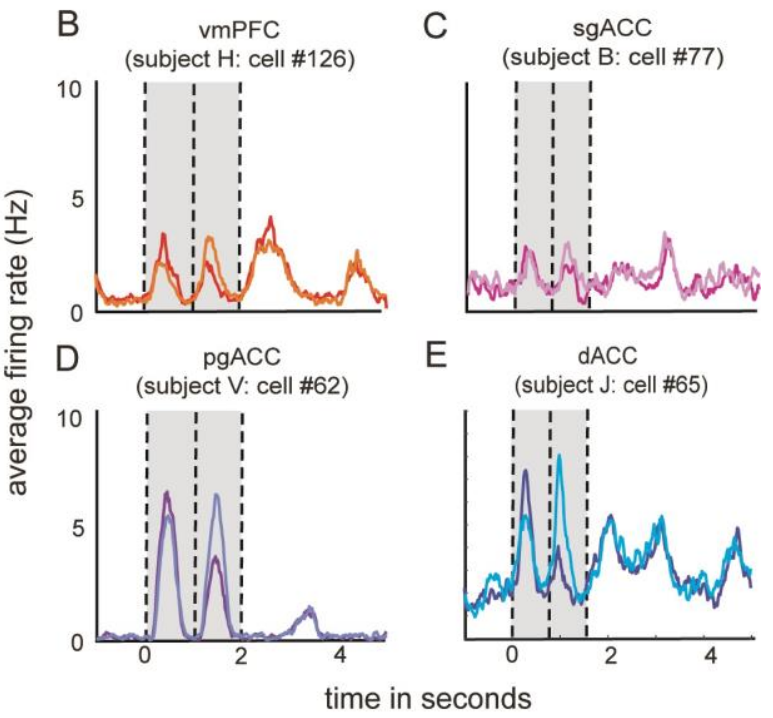

Figure 3. Response latency and traces of average firing rates across all trials drawn from responses of single sample neurons. A. Average latency to maximal firing rates in response to the onset of both offer 1 and offer 2 . Error bars indicate the standard error across trials. B-E. Peri-stimulus time histogram responses of sample neurons with firing rates that are significantly correlated with the expected values of both offers. Traces are grouped by trials on which the value of either offer 1 (darker color) or offer 2 (lighter color) was larger. Traces are smoothed, for display, with a $200 \mathrm{~ms}$ sliding boxcar. Average firing rates are computed in spikes per second. The onset of offer 1 is set to time 0 . The vertical lines indicate the start times of periods in the trial (the onset of offer 1, offer 2, and fixation).

regions encoded the value of offer 2 during epoch 2 (vmPFC: 14.1\%; sgACC: $7.53 \%$; pgACC: 10.98\%; dACC: $14.73 \% ; p<0.01$ in all areas except $\operatorname{sgACC}$ (trending at $p=0.063$ ), binomial test). Finally, neurons encoded the value of offer 1 during epoch 2 (i.e. working memory for 
value, vmPFC: 9.62\%; sgACC: 10.27\%; pgACC: 9.41\%; dACC: $14.73 \% ; p<0.01$ in all areas, binomial test; Figure 4).

205

A vmPFC

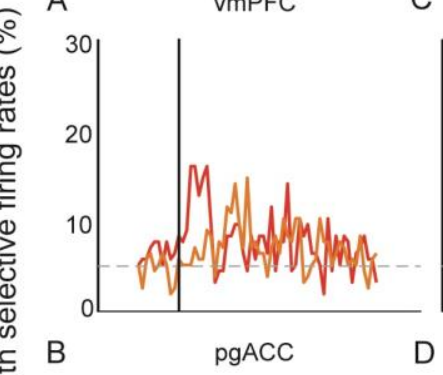

B $\quad$ pgACC

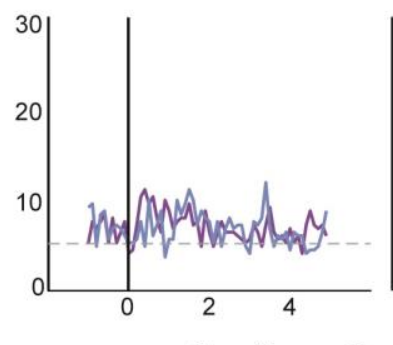

C

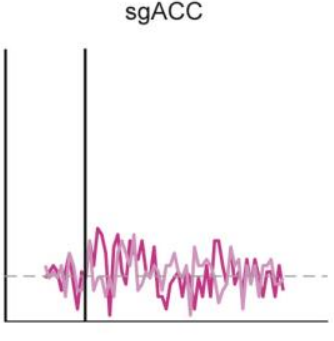

D

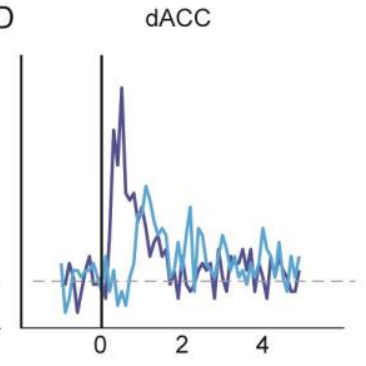

time from offer 1 onset (s)

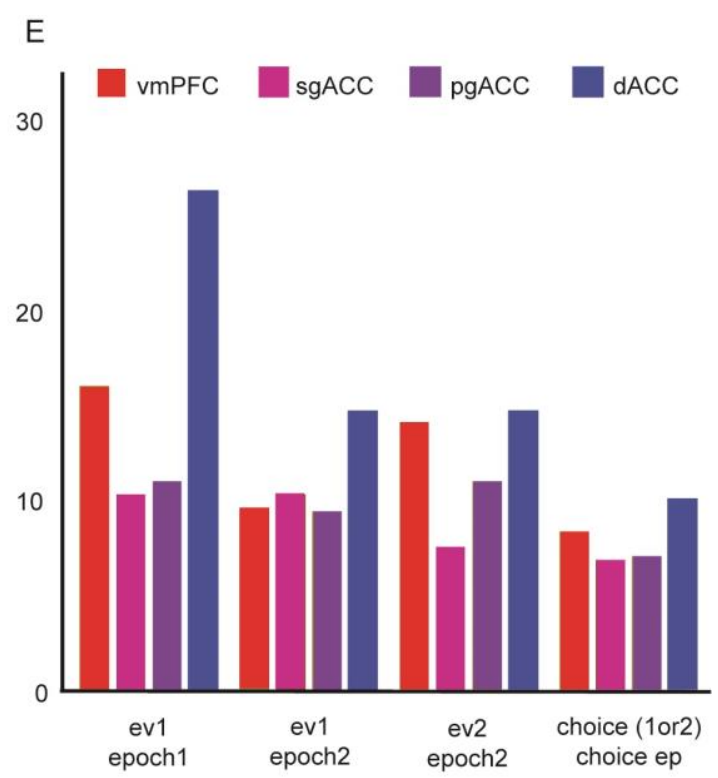

207

208

209

210

211

212

\section{3}

214

215

216

217

218

Figure 4. Selectivity of firing rates to offers and choice. A-D. The proportion of neurons that have firing rates that are correlated with the expected value of offer 1 (darker color) and offer 2 (lighter color) across a six second period during the trial. The onset of offer 1 is set to time 0 . The vertical line indicates the onset of offer 1 . E. A summary of the proportion of neurons selective to offers and choice, within given epochs. Each bar indicates the proportion within a given brain area.

\section{Putative signatures of choice process are found in all four regions}

Feature Integration. We next asked whether each brain region contains a value signal

that reflects the integration of the two features that determine value: probability and magnitude

(Azab and Hayden, 2020). For each brain region, we computed regression weights for each

neuron's normalized (z-scored) firing rates for the two variables. We then examined how those

variables related to each other across the population. A positive correlation between regression

coefficients indicates that both offer features are encoded using a correlated coding scheme. In 
220 other words, it indicates that the population of neurons has thrown out information about the

221 details of the components and has begun to compute an integrated value signal (Blanchard et al.,

222 2015; Azab and Hayden, 2020). We observed a significant positive relationship in all regions ( $p$

$223<0.001$ in all regions; Figure 5A). This result indicates that feature integration is not a unique

224 feature of any region, but instead, is broadly shared across the medial PFC.

Attentional alignment. When attention shifts from one option to another, it is possible that

226 the same population of neurons encodes the new option in the same manner as it encoded the

227 first one, like neurons in visual cortex do for visual stimuli (Hayden and Moreno-Bote, 2018; see

228 also McGinty et al., 2016; Xie et al., 2018). In other words, neurons may act as a flexible filter

229 for value; we have called this principle attentional alignment (as opposed to a labeled line code

230 for value, Hayden and Moreno-Bote, 2018). We next asked whether attentional alignment is a

231 principle shared in all four of our regions. To do so, we regressed normalized firing rates from

232 epoch 1 onto the expected value of offer 1 and regressed normalized firing rates from epoch 2 on

233 the expected value of offer 2 . We then correlated these resulting coefficients. A positive

234 correlation is evidence for attentional alignment. All four regions exhibited significant $(p<0.01)$

235 positive correlations (Figure 5B).

Mutual inhibition. We next asked if there was evidence for direct comparison between

237 offers by principle of mutual inhibition (Strait et al., 2014; Azab and Hayden, 2017). We

238 regressed normalized firing rates from epoch 2 on offer 1 onto responses from epoch 2 on offer

239 2. If the encoding for value 1 and value 2 during the same epoch are anti-correlated, then the

240 encoding of 1 value comes at the expense of the other. it is an indication of the direct comparison

241 of offers and thus a signal of choice. All recorded regions exhibited significant $(p<0.05)$ 
A

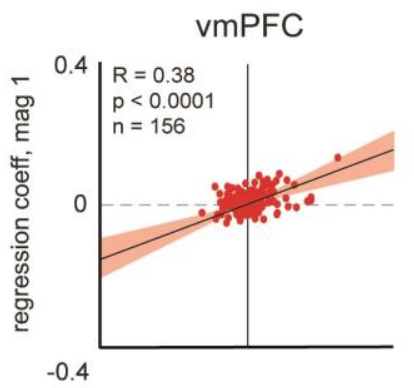

B

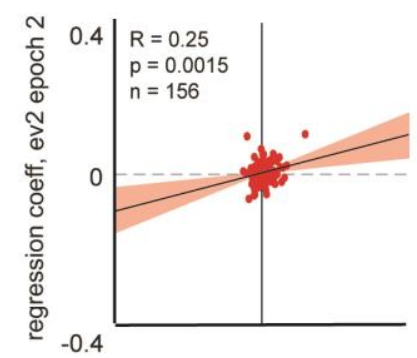

C

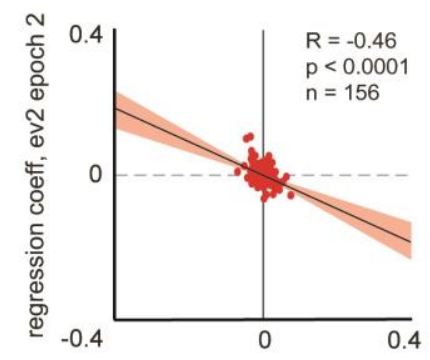

245

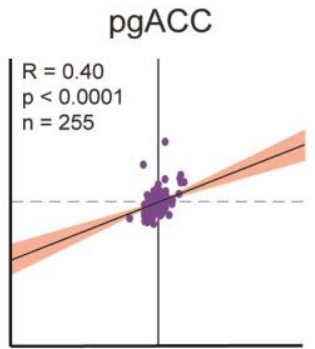

regression coeff, prob 1
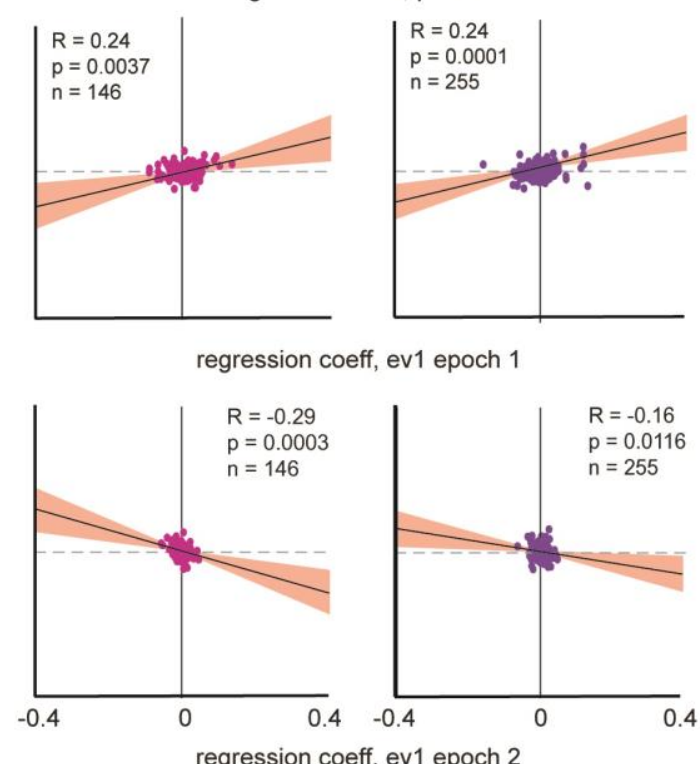

regression coeff, ev1 epoch 2
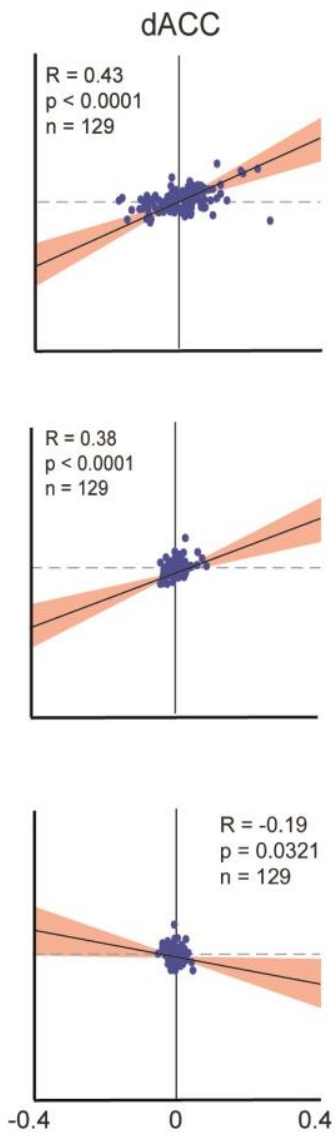

Figure 5. Economic choice functions. A. Feature integration. From left to right: Scatter plots of coefficients from regressing normalized epoch 1 firing rates on the probability of offer 1, against the regression coefficients from the magnitude of offer 1 . The diagonal black line indicates the slope of the correlation between regression coefficients.

The red ribbons indicate the $95 \%$ confidence intervals. B. Attentional alignment. Scatter plots are of coefficients from regressing normalized epoch 1 firing rates on expected value of offer 1 , against the regression coefficients from epoch 2 firing rates on expected value of offer 2. C. Mutual inhibition. Scatter plots are of coefficients from regressing normalized epoch 2 firing rates on the expected value of offer 1, against the regression coefficients from epoch 2 firing rates on the expected value of offer 2 . 
populations of neurons encoding the previously described functions. We repeated the integration,

257 alignment, and inhibition analyses (i.e. the correlations of select regression weights), but now

258 with absolute (i.e. unsigned) values of the regression weights. We have previously shown that

259 such correlations indicate shared or overlapping functional populations (Blanchard et al., 2018).

260 If the (unsigned) strength of encoding of one offer (or feature) is positively correlated with the

261 degree of encoding of the other offer, then the populations associated with encoding the two

262 variables overlap more than expected by chance. We found a significant positive correlation

263 (vmPFC: $\mathrm{R}=0.31$; sgACC: $\mathrm{R}=0.31$; pgACC: $\mathrm{R}=0.44$;ACC: $\mathrm{R}=0.41 ; p<0.001$, all areas)

264 between unsigned betas for the integration function (epoch 1 firing rates regressed on offer 1

265 magnitude and on offer 1 probability). We also found a significantly positive correlation between

266 unsigned betas for all areas (vmPFC: $\mathrm{R}=0.34$; $\operatorname{sgACC}: \mathrm{R}=0.23$; pgACC: $\mathrm{R}=0.33 ; p<0.01$ ),

267 except for dACC $(\mathrm{R}=0.17, p=0.058)$, representing alignment (epoch 1 firing rates regressed on

268 expected value 1 and epoch 2 regressed on expected value 2). Finally, vmPFC $(\mathrm{R}=0.29)$ and

$269 \operatorname{pgACC}(\mathrm{R}=0.25)$ showed significantly positive correlations $(p<0.001$ in both cases) between

270 unsigned betas for inhibition (epoch 2 firing rates on the expected value of each offer). These

271 results indicate that, in most areas and across functions, encoding is mostly supported by the

272 same, or at least overlapping, sets of neurons.

273

274 Intrinsic timescales are longest at the top of the anatomical hierarchy.

These results demonstrate broadly overlapping functions across regions. We next asked

276 whether there is evidence of hierarchy. We first considered intrinsic timescales. Intrinsic

277 timescales are a population-level statistic describing fluctuations in a neuronal signal that are 
278 agnostic to the task and corresponding variables (Murray et al., 2014). Murray and colleagues

279 (2014) proposed that intrinsic fluctuations are a function of increased modulatory strength. They

280 suggested that increased modulation is due to increased recurrent network activity, which in turn

281 increases along a hierarchy. Thus, longer intrinsic timescales would be indicative of increased

282 modulatory strength and, therefore, a higher position along the hierarchy.

283 We estimated and compared the intrinsic timescales of each recorded region from a

284 temporal decay function. We used the decay function to fit the autocorrelation of pre-trial spike

285 data across a range of lags (see Methods). We found an increase of intrinsic timescale that

286 seemed to map a medial prefrontal hierarchy onto a rough ventrodorsal gradient (vmPFC: 109.8

287 ms; sgACC: 152.76 ms; pgACC: 321.85 ms; dACC: 446.51 ms; Figure 6). We confirmed a

288 positive monotonic relationship by correlating the intrinsic timescales with the observed order

289 (1-4) across the areas. The results showed a significant positive correlation between increasing

290 order across the four areas, and the increasing intrinsic timescale $(\mathrm{R}=0.98, p=0.022$, Pearson's

291 correlation; $\mathrm{R}=1, p=0.042$, Spearman's correlation). 
A $\quad$ vmPFC

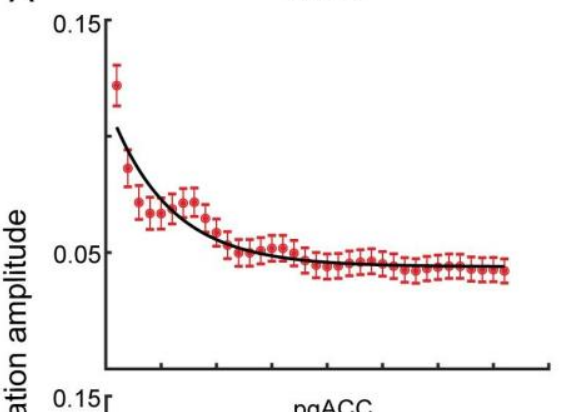

을

过

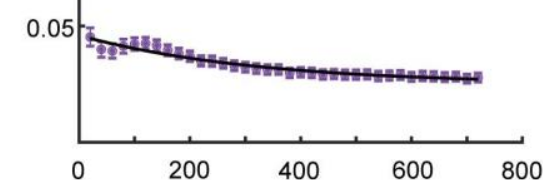

$\mathrm{k} \Delta$ (lag in $\mathrm{ms})$
$\operatorname{sgACC}$

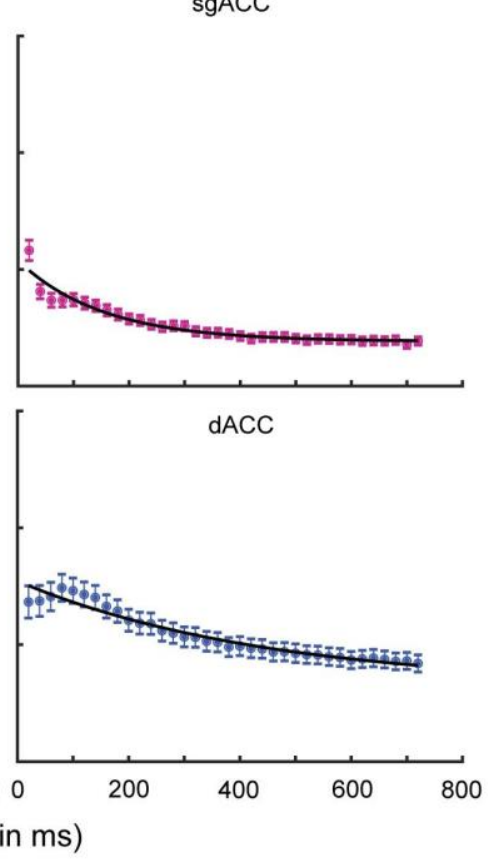

B

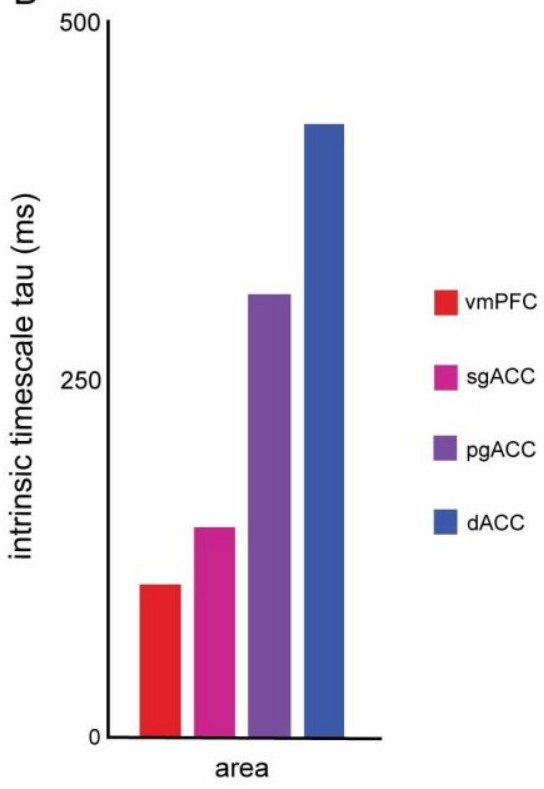

Figure 6. Intrinsic timescales. A. Spike-count autocorrelations calculated at increasing lags for each of the four brain areas. The decay of autocorrelation amplitude with lag was fitted by an exponential decay function with offset (black line). Error bars indicate the variance in autocorrelation amplitude across neurons at a given lag. B. A summary table of the intrinsic timescales, extracted from the exponential decay function. The figure demonstrates a smooth increase of intrinsic timescale along a hierarchy.

\section{Decoding accuracy supports a clear functional hierarchy}

We hypothesized that the accuracy with which expected value and choice can be decoded from firing rate patterns should increase along the observed anatomical hierarchy. Thus, we trained and cross-validated a linear classifier to decode seven binary labels (specifically: high/low expected values for both offers and the difference between them, offer position, choice, and chosen side) from firing rates.

We first looked at how accurately offer 1 value could be decoded from firing rates in epoch 1 (Figure 7A). The classifier decoded whether offer 1 on each trial had greater or lesser value than the mean offer value across trials significantly better than chance $(p<0.0001$, 
binomial test) for all regions: vmPFC (61.4\%), sgACC (63.1\%), pgACC (69.8\%), and dACC

310 (73.7\%). Notably, decoding accuracy increases with hierarchical order $(\mathrm{R}=0.98, p=0.022$,

311 Pearson's correlation; $\mathrm{R}=1, p=0.042$, Spearman's correlation).

We then looked at how accurately offer 1 value could be decoded from firing rates in

7B). The classifier decoded whether offer 1 on each trial had greater or lesser value than the

317 decoding accuracy increases with hierarchical order $(\mathrm{R}=0.95, p=0.049$, Pearson's correlation;

$318 \mathrm{R}=1, p=0.042$, Spearman's correlation).

We next looked at how accurately offer 2 value could be decoded from firing rates in

321 value than the mean offer value across trials significantly better than chance $(p<0.0001$, general increase in decoding accuracy with hierarchical position.

328 from firing rates in epoch 2 (Figure 7D). (This analysis would not make sense in epoch 1

329 because the subject did not know both values yet). The classifier decoded whether offer 
sgACC (62.3\%), pgACC (67\%), and dACC (70.2\%). Decoding accuracy increases with

333

334

335

336

337

338

339

340

341

342

343

344

345

346

347

348

349

350

351

352

353

354

hierarchical order $(\mathrm{R}=0.98, p=0.017$, Pearson's correlation; $\mathrm{R}=1, p=0.042$, Spearman's correlation).

\section{Hierarchical organization of coding of spatial information}

We looked at how accurately the position of offer on the monitor could be decoded from firing rates in epoch 1 (Figure 7E). The classifier decoded whether offer 1 on each trial was on the right or left $(p<0.0001$, binomial test) for all regions: vmPFC $(64.5 \%), \operatorname{sgACC}(69.5 \%)$, $\operatorname{pgACC}(72.6 \%)$, and dACC (77.2\%). Decoding accuracy increases with hierarchical order $(\mathrm{R}=$ 0.996, $p=0.004$, Pearson's correlation; $\mathrm{R}=1, p=0$. 042, Spearman's correlation).

Next, we looked at how accurately the chosen offer (offer 1 or 2 ) could be decoded from firing rates in choice epoch (Figure 7F). Note that this variable is orthogonal to offer side, since we observed essentially no spatial biases in choice. The classifier decoded choice on each trial $(p$ $<0.0001$, binomial test) for all regions: vmPFC (64.2\%), $\operatorname{sgACC}(70.5 \%), \operatorname{pgACC}(70.6 \%)$, and dACC (72.5\%). We determined that decoding accuracy trended toward an increase with hierarchical order $(\mathrm{R}=0.896, p=0.104$, Pearson's correlation; $\mathrm{R}=1, p=0.042$, Spearman's correlation).

We also looked at how accurately the position of the chosen offer on the monitor could be decoded from firing rates in the choice epoch (Figure 7G). The classifier decoded whether the chosen side on each trial was on the right or left $(p<0.0001$, binomial test) for all regions: $\operatorname{vmPFC}(64.7 \%), \operatorname{sgACC}(70 \%), \operatorname{pgACC}(70.4 \%)$, and dACC (75.6\%). We determined that decoding accuracy increases with hierarchical order $(\mathrm{R}=0.96, p=0.041$, Pearson's correlation; $\mathrm{R}=1, p=0.042$, Spearman's correlation). 
We also looked at how accurately the position of the chosen offer on the monitor could

357 classifier decoded whether the chosen side on each trial was on the right or left $(p<0.0001$,

358 binomial test) for all regions: vmPFC (69.3\%), sgACC (72.4\%), pgACC (70.8\%), and dACC

$359(83 \%)$. We determined that decoding accuracy does not significantly increase with hierarchical

360 order $(\mathrm{R}=0.82, p=0.179$, Pearson's correlation; $\mathrm{R}=0.8, p=0.167$, Spearman's correlation).

361 While this pattern was not significant, there still appears to be a general increase in decoding

362 accuracy with hierarchical position.

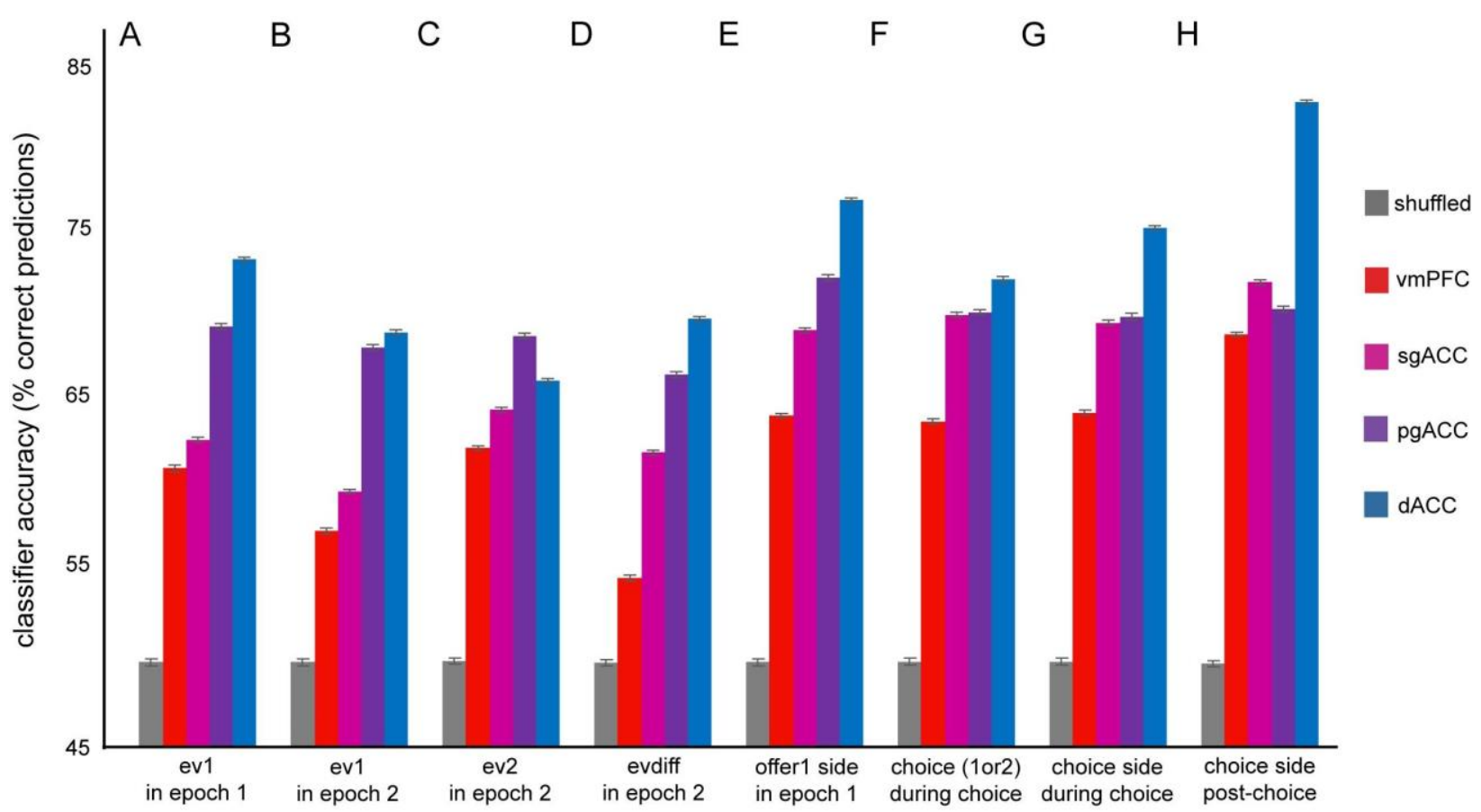

Figure 7. Decoding analysis. A-H. Summary of classification accuracies for each of the given labels. In a given epoch, a linear classifier was trained to identify the value of a binary label (indicated on the X-axis). The accuracy of the trained model was tested by cross-validation, to predict the label value on novel data (indicated on the Y-axis). Accuracy of a model trained on randomly shuffled data are indicated by the grey bar. Error bars represent the standard error over the variance across cross-validations. 


\section{DISCUSSION}

Here we examined neuronal correlates of multiple elements of economic choice in four

373 medial prefrontal cortex regions. Confirming and extending our previous results, we find that

374 these regions show largely similar value-related signals (Strait et al., 2014; Azab and Hayden,

375 2018). Indeed, by none of the measures we chose did these regions differ qualitatively. This

376 result suggests that the regions do not have conspicuous qualitative differences along the

377 dimensions we studied, but leaves open the possibility that they differ quantitatively. Our major

378 novel finding is that, by several measures, the regions appear to be organized hierarchically.

379 First, eight basic task variables are consistently more decodable later in the hierarchy. These

380 include both abstract (economic) and spatial variables. Second, intrinsic timescale is longer later

381 in the hierarchy. Overall, our results are consistent with the idea that the four regions serve as

382 part of a roughly ventral-to-dorsal functional gradient that gradually transforms neural encodings

383 (Yoo and Hayden, 2018).

The idea that prefrontal regions have a largely hierarchical organization was pioneered by

385 Fuster, who proposed a functional gradient from the sensory to the motor areas, and involving

386 "association cortex" between them (Fuster, 2001). Although he (like many subsequent thinkers)

387 was mainly focused on the lateral prefrontal cortex, the same logic may extend to medial areas.

388 However, the most logical organization of such areas is not obvious, either anatomically or

389 functionally. There are many possibilities. Primarily using anatomical connectivity patterns,

390 Price and colleagues classify all four of our recorded regions in his "medial network," which

391 they propose are responsible for visceromotor functions, and contrasting with the "orbital

392 network," responsible for sensory functions (Carmichael and Price, 1994; Carmichael and Price,

393 1996; Ongur and Price, 2000). 

take a somewhat different view. For them, areas 25, 24, and 32, as relatively undifferentiated cingulate cortex, are all placed in a similar, low position in a mediodorsal hierarchy. Area 14,

397 split between the mediodorsal and basoventral trends, occupies a somewhat higher position in the

398 hierarchy. Our results (although we interpret them differently with respect to a hierarchy) are not 399 necessarily inconsistent with such a framework, as increased decodability and timescales may 400 simply be a hallmark of less-differentiated PFC regions.

Alternatively, topology would suggest possible ventrodorsal $(14 \rightarrow 25 \rightarrow 32 \rightarrow 24)$ or genu-

402 adhering $(25 \rightarrow 14 \rightarrow 32 \rightarrow 24)$ hierarchies. The first one is consistent with the idea that OFC

403 (Price's orbital network) serves as the entryway for economic information to the prefrontal

404 cortex, and area 14 as its next station (Rushworth et al., 2011; Noonan et al., 2010). Our work

405 supports the ventrodorsal hypothesis most strongly, thereby offering the first

406 electrophysiological evidence for one specific medial prefrontal hierarchy. One prediction of this

407 hierarchy is that medial area 9 (dorsomedial prefrontal cortex) should be one step above the

408 recorded areas in our analyses (cf. Schall et al., 2002). We might also expect that sensory choice

409 information is received by orbitofrontal cortical area 13, and then relayed to the medial

410 prefrontal cortex; thus we would expect area 13 to be below the recorded areas in this hierarchy,

411 to have shorter intrinsic timescales, and to have less decodable information.

412 Our results suggest that these four regions have largely overlapping functions in the

413 domain of economic choice. Notably, our results do not imply that these regions have identical

414 functions, nor that their differences are solely quantitative. Indeed, there is plentiful evidence that

415 these regions have important qualitative differences (Bush et al, 2000; Vogt and Laureys, 2005;

416 Van Holstein and Floresco, 2020). To give an example, in a social aggression paradigm, 
417 activation of the ventral medial prefrontal cortex correlates with skin conductance response,

418 perhaps reflecting its strong interactions with hypothalamus and periaqueductal gray, while

419 activation of the dorsal medial prefrontal cortex is more cognitive in nature (Lotze et al., 2007).

420 Our results do not challenge or invalidate such categorical functional differences. Rather, they

421 suggest that these regions have qualitative differences in some domains and quantitative

422 differences in at least one domain, the domain of economic choice. Indeed, our results do point

423 to a potential limitation to much of traditional functional neuroanatomy. Much of that work is

424 focused exclusively on identifying the unique contributions of particular regions. While that

425 work is critically important, it necessarily ignores the kinds of brain functions that are not

426 uniquely implemented by specific regions. We believe that economic choice is one such function

427 (Yoo and Hayden, 2018).

428 Nonetheless, a broad reading of the electrophysiological literature highlights that many

429 functions have traces that are quite similar in multiple regions (Hunt and Hayden, 2017). Many

430 scholars draw a distinction between sensory areas, for which a strong modularity case can be

431 made, and “association areas". For example, as Prinz (2006) points out, even Fodor, a great

432 advocate of modularity, was more willing to consider distributed function outside of sensory and

433 motor regions (Fodor, 1983). Likewise, Uttal (2001) identifies Olds' work on classical (trace)

434 conditioning (1972), which shows that correlates of trace conditioning can be found in nearly

435 every part of the rat brain. 


\section{METHODS}

439 were designed and conducted in compliance with the Public Health Service's Guide for the Care

440 and Use of Animals. Four male rhesus macaques (Macaca mulatta) served as subjects for both

441 tasks. A small prosthesis head fixation was used. Animals were habituated to laboratory

442 conditions and then trained to perform oculomotor tasks for liquid rewards. We place a Cilux

443 recording chamber (Crist Instruments) over the area of interest (see Behavioral tasks for

444 breakdown). We verified positioning by magnetic resonance imaging with the aid of a Brainsight

445 system (Rogue Research). Animals received appropriate analgesics and antibiotics after all

446 procedures. Throughout both behavioral and physiological recording sessions, we kept the

447 chamber with regular antibiotic washes and we sealed them with sterile caps.

$448 \quad$ Recording sites. We approached our brain regions through standard recording grids (Crist

449 Instruments) guided by a micromanipulator (NAN Instruments).

We defined vmPFC 14 as lying within the coronal planes situated between 29 and 44

$451 \mathrm{~mm}$ rostral to the interaural plane, the horizontal planes situated between 0 and $9 \mathrm{~mm}$ from the

452 brain's ventral surface, and the sagittal planes between 0 and $8 \mathrm{~mm}$ from the medial wall (Figure

453 2B). These coordinates correspond to area 14 in Paxinos et al. (2008).

454 We defined sgACC 25 as lying within the coronal planes situated between 24 and $36 \mathrm{~mm}$

455 rostral to interaural plane, the horizontal planes situated between 17.33 and $25.12 \mathrm{~mm}$ from the

456 brain's dorsal surface, and the sagittal planes between 0 and $5.38 \mathrm{~mm}$ from medial wall (Figure

457 2B). Our recordings were made from central regions within these zones, which correspond to

458 area 25 in Paxinos et al. (2008). 
We defined pgACC 32 as lying with the coronal planes situated between 30.90 and 40.10

460

461

462

463

464

465

466

467

468

469

470

471

472

473

474

475

476

477

478

479 isolated on a Plexon system (Plexon, Dallas, TX) or Ripple Neuro (Salt Lake City, UT). Neurons

480

481

$\mathrm{mm}$ rostral to the interaural plane, the horizontal planes situated between 7.30 and $15.50 \mathrm{~mm}$

from the brain's dorsal surface, and the sagittal planes between 0 and $4.5 \mathrm{~mm}$ from the medial

wall (Figure 2B). Our recordings were made from central regions within these zones, which

correspond to area 32 in Paxinos et al. (2008).

We defined dACC 24 as lying within the coronal planes situated between 29.50 and

$34.50 \mathrm{~mm}$ rostral to interaural plane, the horizontal planes situated between 4.12 to $7.52 \mathrm{~mm}$

from the brain's dorsal surface, and the sagittal planes between 0 and $5.24 \mathrm{~mm}$ from medial wall

(Figure 2B). Our recordings were made from central regions within these zones, which

correspond to area 24 in Paxinos et al. (2008).

We confirmed recording location before each recording session using our Brainsight

system with structural magnetic resonance images taken before the experiment. Neuroimaging

was performed at the Rochester Center for Brain Imaging on a Siemens 3T MAGNETOM Trio

Tim using $0.5 \mathrm{~mm}$ voxels. We confirmed recording locations by listening for characteristic

sounds of white and gray matter during recording, which in all cases matched the loci indicated

by the Brainsight system with an error of $\sim 1 \mathrm{~mm}$ in the horizontal plane and $\sim 2 \mathrm{~mm}$ in the $\mathrm{z}$ -

direction.

Electrophysiological techniques. Either single (FHC; starting impedance $4 \mathrm{M} \Omega$ ) or multi-

contact electrodes (V-Probe, Plexon) were lowered using a microdrive (NAN Instruments) until

waveforms between one and three neuron(s) were isolated. Individual action potentials were

were selected for study solely on the basis of the quality of isolation; we never preselected based

on task-related response properties. All collected neurons for which we managed to obtain at 
482 least 300 trials were analyzed; no neurons that surpassed our isolation criteria were excluded

483 from analysis. eye-monitoring camera system (SR Research). Stimuli were controlled by a computer running Matlab (Mathworks) with Psychtoolbox and Eyelink Toolbox. Visual stimuli were colored

487 rectangles on a computer monitor placed $57 \mathrm{~cm}$ from the animal and centered on its eyes (Fig.

488 1A). A standard solenoid valve controlled the duration of juice delivery. Solenoid calibration was 489 performed daily. structure. For the neuronal recordings in vmPFC, subjects $B$ and $H$ performed the risky choice

492 task; and for dACC and sgACC, subjects B and $J$ performed the token risky choice task (Fig.

$4932 C$ ). Both tasks made use of vertical rectangles indicating reward amount and probability. We

494 have shown in a variety of contexts that method provides reliable communication of abstract

495 concepts such as reward, probability, delay, and rule to monkeys (Hayden et al., 2009; Blanchard

496 and Hayden 2014; Sleezer et al., 2016; Mehta et al., 2019).

497 Risky choice task (vmPFC and pgACC 32). All tasks were based on a standardized

498 general structure for gambling tasks (Hayden et al, 2010; Heilbronner and Hayden, 2013;

499 Heilbronner and Hayden, 2016; Heilbronner, 2017). The task presented two offers on each trial.

500 A rectangle 300 pixels tall and 80 pixels wide represented each offer $\left(11.35^{\circ}\right.$ of visual angle tall

501 and $4.08^{\circ}$ of visual angle wide; Fig. 2A). Two parameters defined gamble offers, reward size and

502 probability. Two portions divided each gamble rectangle, one red and the other either grey, blue,

503 or green. The size of the color portions signified the probability of winning a small (125 $\mu$ l),

504 medium (mean $165 \mu \mathrm{l}$ ), or large reward (mean $240 \mu \mathrm{l}$ ), respectively. We drew a uniform 
distribution between 0 and $100 \%$ for these probabilities. Red colored the rest of the bar; the size of the red portion indicated the probability of no reward. Offer types were selected at random with a $43.75 \%$ probability of blue (medium magnitude) gamble, a $43.75 \%$ probability of green

508 (high magnitude) gambles, and a $12.5 \%$ probability of gray options (safe offers).

510 the right. We randomized the sides of the first and second offer (left and right). Each offer

511 appeared for $400 \mathrm{~ms}$ and was followed by a 600-ms blank period. After the offers were presented

512 separately, a central fixation spot appeared and the monkey fixated on it for $100 \mathrm{~ms}$. Following

513 this, both offers appeared simultaneously and the animal indicated its choice by shifting gaze to

514 its preferred offer and maintaining fixation on it for $200 \mathrm{~ms}$. Failure to maintain gaze for $200 \mathrm{~ms}$

515 did not lead to the end of the trial but instead returned the monkey to a choice state; thus

516 monkeys were free to change their mind if they did so within $200 \mathrm{~ms}$ (although in our

517 observations, they seldom did so). Following a successful 200-ms fixation, the trial immediately

518 resolved the gamble and delivered the reward. We considered trials that took $\sim 7 \mathrm{~s}$ as inattentive

519 trials and we did not include them in the analyses (this removed $\sim 1 \%$ of trials). Outcomes that

520 yielded rewards were accompanied by a visual cue: a white circle in the center of the chosen

521 offer. All trials were followed by an 800-ms intertrial interval with a blank screen.

$522 \quad$ Token risky choice task ( $\operatorname{sgACC} 25$ and dACC 24). Another similarly structured gambling

523 task, where gambles each had two potential outcomes, wins or losses in terms of "tokens"

524 displayed on screen as cyan circles. A small reward $(100 \mu l)$ was administered concurrently with

525 gamble feedback on each trial, regardless of gamble outcome. Trials in which the monkey

526 accumulated six or more tokens triggered an extra "jackpot" epoch in which a very large reward

$527(300 \mu 1)$ was administered (Fig. 2C). 
Behavioral analysis. To confirm the statistical validity of the behavioral results, we first

529

530

531

532

533

534

535

536

537

538

539

540

541

542

543

544

545

546

547 calculated the average firing rate for each neuron, for each $20 \mathrm{~ms}$ bin, across trials. Then, for

548 each neuron, we determined how much time (in ms) passed for a given neuron to reach its peak

549 firing rate for the epoch. Finally, we calculated the average latency to peak firing rate across all

550 neurons in the region. 
Intrinsic timescales. To measure intrinsic timescales, we followed similar steps described in Murray, et al. (2014). We isolated a 2-second time window preceding the onset of offer 1, to remain independent of trial variables. Using a $20 \mathrm{~ms}$ sliding window, we then computed the

554 autocorrelation for the 2 second window with a given lag $k \Delta$ between time $i$ and time $j$, where $555 k \Delta=|i-j|$. The lag ranged from $20 \mathrm{~ms}$ and $720 \mathrm{~ms}$. We then determined that the autocorrelation 556 decay in each structure could be well-fit by an exponential decay function:

$R(k \Delta)=A[\exp (-k \Delta / \tau)+B]$

560 where $A=$ the amplitude of the autocorrelation, $k \Delta=$ the lag, $\tau=$ intrinsic timescale, and $B=$ the

561 offset to account for long timeframes outside of the measured window. This formula follows

562 what was described by Murray, et al. 2014.

Decoding analysis. We built a pseudo-population of pseudo-trials. First, we isolated each

564 epoch and collapsed the firing rates for each trial into an average for the $500 \mathrm{~ms}$ period. Then, we

565 separated the data set for each neuron by the given label (1st offer and second; left offer and

566 right; offer 1 position left or right; offer 2 position left or right; offer 1 value higher or lower than

567 the mean value; and offer 2 value higher or lower than the mean value). We randomly selected

5681000 samples for each neuron resulting in $2 \mathrm{n} \mathrm{X} 1000$ matrices (one for each label level), where

$569 \mathrm{n}$ represented the number of neurons recorded from each region. This constituted the pseudo-

570 population or pseudo-trials. To execute the decoder, each matrix was split in half and

571 concatenated with the half from the other label. We used one of these matrices to train a binary

572 support vector machine, the other was used for cross-validation. We used the trained model to

573 predict the binary label for each pseudo-trial in the cross-validation set. We then compared 
574 predicted outcome to the known choice outcome and an accuracy rate was calculated across

575 pseudo-trials. This process was repeated 100 times for each target structure and for each of the

576 given labels and epochs to get a distribution of accuracy rates. Thus, the standard error of the

577 mean, used in displaying the error bars, represents the standard error over the variance of the

578 cross-validations. Additionally, the exact process was repeated on randomly shuffled data, to

579 confirm that expected prediction accuracy was $50 \%$ when randomized. 


\section{References}

582 Anderson, M. L. (2007). Evolution of cognitive function via redeployment of brain areas.

Neuroscientist, 13(1). https://doi.org/10.1177/1073858406294706

584 Azab, H., \& Hayden, B. Y. (2017). Correlates of decisional dynamics in the dorsal anterior cingulate cortex. PLoS Biology, 15(1). https://doi.org/10.1371/journal.pbio.2003091

Azab, H., \& Hayden, B. Y. (2018). Correlates of economic decisions in the dorsal and subgenual anterior cingulate cortices. European Journal of Neuroscience, 47(8), 979-993. https://doi.org/10.1111/ejn.13865

Azab, H., \& Hayden, B. Y. (2020). Partial integration of the components of value in anterior cingulate cortex. Behavioral Neuroscience, 134(4), 296.

591 Badre, D. (2008). Cognitive control, hierarchy, and the rostro-caudal organization of th prefrontal lobes. Trends in Cognitive Sciences, 12(5), 193-200.

593 Balasubramani, P. P., Moreno-Bote, R., \& Hayden, B. Y. (2018). Using a simple neural network to delineate some principles of distributed economic choice. Frontiers in Computational Neuroscience, 12(22). https://doi.org/10.3389/fncom.2018.00022

596 Barbas, H. \& Pandya, D. N. (1989). Architecture and intrinsic connections of the prefrontal cortex in the rhesus monkey. Journal of Comparative Neurology, 286(3). https://doi.org/10.1002/cne.902860306 model. Journal of Machine Learning Research, 3, 1137-1155.

601 Blanchard, T. C., \& Hayden, B. Y. (2014). Neurons in dorsal anterior cingulate cortex signal postdecisional variables in a foraging task. Journal of Neuroscience, 34(2), 646-655. https://doi.org/10.1523/JNEUROSCI.3151-13.2014 
604 Blanchard, T. C., Hayden, B. Y., \& Bromberg-Martin, E. S. (2015). Orbitofrontal cortex uses distinct codes for different choice attributes in decisions motivated by curiosity. Neuron, $85,602-614$.

Blanchard, T. C., Piantadosi, S. T., \& Hayden, B. Y. (2018). Robust mixture modeling reveals category-free selectivity in reward regions neuronal ensembles. Journal of Neurophysiology, 119(4).

Bush, G., Luu, P., \& Posner, M. I. (2000) Cognitive and emotional influences in anterior cingulate cortex. Trends in Cognitive Sciences, 4(6).

Cai, X. \& Padoa-Schioppa, C. (2014). Contributions of orbitofrontal and lateral prefrontal cortices to economic choice and the Good-to-Action transformation. Neuron, 81(5).

614 Carmichael, S. T. \& Price, J. L. (1994). Architecture subdivision of the orbital and medial prefrontal cortex in the macaque monkey. Journal of Comparative Neurology, 346(3). https://doi.org/10.1002/cne.903460305

Carmichael, S. T., \& Price, J. L. (1995). Limbic connections of the orbital and medial prefrontal cortex in macaque monkeys. Journal of Comparative Neurology, 363(4), 615-641.

619 Carmichael, S. T., \& Price, J. L. (1996). Connectional networks within the orbital and medial prefrontal cortex of macaque monkeys. Journal of Comparative Neurology, 371(2), 179-

622 Cisek, P. (2012). Making decisions through a distributed consensus. Current Opinion in Neurobiology, 22(6).

624 Cisek, P. \& Kalaska, J. (2010). Neural mechanisms for interacting with a world full of action choices. Annual Review of Neuroscience, 33. 
David, S. V., \& Hayden, B. Y. (2012). Neurotree: A collaborative, graphical database of the academic genealogy of neuroscience. PloS one, 7(10), e46608.

DiCarlo, J. J., \& Cox, D. D. (2007). Untangling invariant object recognition. TRENDS in Cognitive Neuroscience, 11(8).

DiCarlo, J. J., Zoccolan, D., \& Rust, N. C. (2012) How does the brain solve visual object recognition? Neuron: Perspective, 73. strategy on response time during complex value-based learning and choice. PLOS One, $13(5)$.

Farashahi, S., Donahue, C. H., Hayden, B. Y., Lee, D., \& Soltani, A. (2019). Flexible combination of reward information during choice under certainty. Nature Human Behavior, 3(11).

638 Felleman, D. J., \& Van Essen, D. C. (1991). Distributed hierarchical processing in the primate cerebral cortex. Cerebral Cortex, 1, 1-47. https://doi.org/10.1093/cercor/1.1.1

640 Fodor, J. A. (1983). The Modularity of the Mind: An essay on faculty psychology. Cambridge, 641 MA and London, England, UK: The MIT Press.

642 Freedman, L. J., Insel, T. R., \& Smith, Y. (2000). Subcortical projections of area 25 (subgenual cortex) of the macaque monkey. Journal of Comparative Neurology, 421(2), 172-188.

644 Fuster, J. M. (1990). Prefrontal cortex and bridging of temporal gaps in the perception-action cycle. Annals of the New York Academy of Sciences, 608(1).

646 Fuster, J. M. (2001). The prefrontal cortex - An update: Time is of the essence. Neuron, 30, 319- 
Haber, S. H. \& Behrens, T. E. J. (2014). The neural network underlying incentive-based 83(5).

Haber, S. H. \& Knutson, B. (2010). The reward circuit: Linking primate anatomy and human imaging. Neuropsychopharmacology, 35.

Hare, T. A., Schultz, W., Camerer, C. F., O’Doherty, J. P., \& Rangel, A. (2011). Transformation of stimulus value signals into motor commands during simple choice. PNAS, 108(44), $18120-18125$.

Hauser, T. U., Hunt, L. T., Iannaccone, R., Walitza, S., Brandeis, S., Brandeis, D., Brem, S., \& Dolan R. J. (2015). Temporally dissociable contributions of human medial prefrontal subregions to reward-guided learning. Journal of Neuroscience, 35(32). DOI: Frontiers in neuroscience, 4, 166.

Hayden, B. Y., \& Moreno-Bote, R. (2018). A neuronal theory of sequential economic choice. cingulate cortex. Science, 324(5929), 948-950. features. Current opinion in behavioral sciences, 16, 23-29. in rhesus monkeys. Frontiers in Neuroscience, 7(7). https://doi.org/10.3389/fnins.2013.00007 
671 Heilbronner, S. R., \& Hayden, B. Y. (2016). The description-experience gap in risky choice in nonhuman primates. Psychonomic Bulletin \& Review, 23(2), 593-600.

673 Hunt, L. T., Behrens, T. E. J., Hosokawa, T., Wallis, J. D., \& Kennerly, S. W. (2015). Capturing the temporal evolution of choice across the prefrontal cortex. eLife, 4. doi:

676 Hunt, L. T., \& Hayden, B. Y. (2017). A distributed, hierarchical and recurrent framework for reward-based choice. Nature Reviews Neuroscience, 18(3), 172-182. https://doi.org/10.1038/nrn.2017.7

679 Hunt, L. T., Kolling, N., Soltani, A., Woolrich, M. W., Rushworth, M. F. S., \& Behrens, T. E. J. (2012). Mechanisms underlying cortical activity during value-guided choice. Nature Neuroscience, 15, 470-476. https://doi.org/10.1038/nn.3017 computations across prefrontal cortex. Nature Neuroscience, 21(10), 1471-1481. https://doi.org/10.1038/s41593-018-0239-5

Kable, J. W., \& Glimcher, P. W. (2009). The Neurobiology of Decision: Consensus and Controversy. Neuron, 63(6). https://doi.org/10.1016/j.neuron.2009.09.003

688 Kennerley, S. W. \& Wallis, J. D. (2009). Reward-dependent modulation of working memory in lateral prefrontal cortex. Journal of Neuroscience, 29(10).

690 Koechlin, E., Danek, A., Burnod, Y., \& Grafman, J. (2002). Medial prefrontal and subcortical 691 mechanisms underlying the acquisition of motor and cognitive action sequences in humans. Neuron, 35(2). 
693 Koechlin, E., Ody, C., \& Kouneiher, F. (2003). The architecture of cognitive control in the human prefrontal cortex. Science, 30(5648).

Lotze, M., Veit, R., Anders, S., \& Birbaumer, N. (2007). Evidence for different role of the ventral and dorsal medial prefrontal cortex for social reactive aggression: An interactive

Maunsell, J. H. \& Van Essen, D. C. (1983). Functional properties of neurons in middle temporal visual area of the macaque. I. Selectivity for stimulus direction, speed, and orientation. Journal of Neurophysiology, 49(5). https://doi.org/10.1152/jn.1983.49.5.1127

McGinty, V. B., Rangel, A., \& Newsome, W. T. (2016). Orbitofrontal Cortex Value Signals Depend on Fixation Location during Free Viewing. Neuron, 90(6). https://doi.org/10.1016/j.neuron.2016.04.045

Mehta, P. S., Tu, J. C., LoConte, G. A., Pesce, M. C., \& Hayden, B. Y. (2019). Ventromedial prefrontal cortex tracks multiple environmental variables during search. Journal of Neuroscience, 39(27), 5336-5350.

Murray, J. D., Bernacchia, A., Freedman, D. J., Romo, R., Wallis, J. D., Cai, X., .. Wang, X. J. (2014). A hierarchy of intrinsic timescales across primate cortex. Nature Neuroscience,

713 Noonan, M. P., Walton, M. E., Behrens, T. E. J., Sallet, B. J., Buckley, M. J., \& Rushworth, F. S. 17(12), 1661-1663. https://doi.org/10.1038/nn.3862

Nee, D. E., Jahn, A., \& Brown, J. W. (2013). Prefrontal cortex organization: Dissociating effects (2010). Separate value comparison and learning mechanisms in macaque medial and lateral orbitofrontal cortex. PNAS, 107(47). 
716 Öngür, D., \& Price, J. L. (2000). The organization of networks within the orbital and medial 717 prefrontal cortex of rats, monkeys and humans. Cerebral cortex, 10(3), 206-219.

718 Padoa-Schioppa, C. (2011). Neurobiology of Economic Choice: A Good-Based Model. Annual

719 Review of Neuroscience, 34(1), 333-359. https://doi.org/10.1146/annurev-neuro-061010-

$720 \quad 113648$

721 Passingham, R. E., \& Wise, S. P. (2015). The Neurobiology of the Prefrontal Cortex: Anatomy

$722 \quad$ and the Origin of Insight. Oxford Scholarship Online. https://doi.org/10.1093/acprof:osobl/9780199552917.001.0001

724 Paxinos, G., Petrides, M., Huang, X., \& Toga, A. W. (2008). The rhesus monkey brain in $725 \quad$ stereotaxic coordinates. Elsvier Science.

726 Pennington, J., Socher, R., and Manning, C. D. (2014). Glove: Global vectors for word representation. Proceedings of the Empirical Methods in Natural Language Processing (EMNLP 2014), 12.

729 Pirrone, A., Azab, H., Hayden, B. Y., Stafford, T., \& Marshall, J. A. (2018). Evidence for the speed-value trade-off: Human and monkey decision making is magnitude sensitive.

732 Plaut, D. C. (1995). Double dissociation without modularity: Evidence from connectionist neuropsychology. Journal of Clinical and Experimental Neuropsychology.

735 Prinz, J. J. (2006). Is the mind really modular? Contemporary Debates in Cognitive Science.

736 Prinz, A. A. (2006). Insights from models of rhythmic motor systems. Current Opinion in 737 Neurobiology, 16(6). https://doi.org/10.1016/j.conb.2006.10.001 
738 Rangel, A., Camerer, C., \& Montague, P. R. (2008). A framework for studying the neurobiology of value-based decision making. Nature Reviews Neuroscience, 9, 545-556. https://doi.org/10.1038/nrn2357

Rudebeck, P. H. \& Murray, E. A. (2014). The orbitofrontal oracle: Cortical mechanisms for the prediction and evaluation of specific behavioral outcomes. Neuron, 84(6).

Rushworth, M. F. S., Behrens, T. E. J., Rudebeck, P. H., \& Walton, M. E. (2007). Contrasting roles for cingulate and orbitofrontal cortex in decisions and social behaviour. Trends in Cognitive Sciences, 11(4).

Rushworth, M. F. S., Noonan, M. A. P., Boorman, E. D., Walton, M. E., \& Behrens, T. E. 70(6), 1054-1069. https://doi.org/10.1016/j.neuron.2011.05.014 frontal lobes. Neuron, 36(2), 309-322.

Siegel, M., Buschman, T., \& Miller, E. (2015). Cortical information flow during flexible

Sleezer, B. J., Castagno, M. D., \& Hayden, B. Y. (2016). Rule encoding in orbitofrontal cortex and striatum guides selection. Journal of Neuroscience, 36(44), 11223-11237.

755 Strait, C. E., Blanchard, T. C., \& Hayden, B. Y. (2014). Reward value comparison via mutual inhibition in ventromedial prefrontal cortex. Neuron, 82(6), 1357-1366. https://doi.org/10.1016/j.neuron.2014.04.032

758 Strait, C. E., Sleezer, B. J., \& Hayden, B. Y. (2015). Signatures of value comparison in ventral striatum neurons. PLoS Biology, 13(6). https://doi.org/10.1371/journal.pbio.1002173 
Strait, C. E., Sleezer, B. J., Blanchard, T. C., Azab, H., Castagno, M. D., \& Hayden, B. Y. (2016). Neuronal selectivity for spatial positions of offers and choices in five reward regions. Journal of Neurophysiology, 115, 1098-1111. https://doi.org/10.1152/jn.00325.2015

764 Tsujimoto, S., Genovesio, A., \& Wise, S. P. (2009). Monkey orbitofrontal cortex encodes response choices near feedback time. Journal of Neuroscience, 29(8), 2569-2574.

Uttal, W. R. (2001). The new phrenology: the limits of localizing cognitive processes in the brain. Cambridge, MA: MIT Press.

Van Holstein, M. \& Floresco, S. B. (2020). Dissociable roles for the ventral and dorsal medial prefrontal cortex in cue-guided risk/reward decision making. Neuropsychopharmacology, 45, 683-693.

771 Vogt, B. A., Rosene, D. L., \& Pandya, D. N. (1979). Thalamic and cortical afferents differentiate anterior from posterior cingulate cortex in the monkey. Science, 204(4389), 205-207.

773 Vogt, B. A. \& Laureys, S. (2005). PosteriorcCingulate, precuneal \& retrosplenial cortices: Cytology \& components of the neural network correlates of consciousness. Progress in Brain Research, 150, 205-217.

776 Wallis, J. D. \& Kennerly, S. W. (2010). Heterogeneous reward signals in prefrontal cortex.

777 Current Opinion in Neurobiology, 20(2).

778 Xie, Y., Nie, C., \& Yang, T. (2018). Covert shift of attention modulates the value encoding in the orbitofrontal cortex. ELife. https://doi.org/10.7554/eLife.31507

780 Yoo, S. B. M., \& Hayden, B. Y. (2018). Economic Choice as an Untangling of Options into Actions. Neuron, 99(3), 434-447. https://doi.org/10.1016/j.neuron.2018.06.038 